\title{
An algorithm for topology optimization of gear reducer housing elements
}

\author{
Stoyan D. Slavov ${ }^{1, *}$, and Mariya Iv. Konsulova-Bakalova ${ }^{1}$ \\ ${ }^{1}$ Technical University of Varna, Department of Manufacturing Technologies and Machine Tools, 9010 Varna, Bulgaria
}

\begin{abstract}
In recent years, topology optimization methods are becoming more widely used in many engineering fields, and they are already being successfully integrated at the design stage of the different types of products. An active field of research in this area is the definition of appropriate constraints in topology optimization models in order to facilitate the production of the optimized objects. An algorithm for topology optimization of housing elements from gear reducers by using the capabilities of CAD-CAE topology optimization software is presented in the current work. The purposed algorithm is taking into account the resulting loads during operation of the reducer, the geometrical and manufacturing constraints of the production process of these housing elements. Obtained results from conducted Taguchi experimental study to investigate the impact of some topology optimization control parameters over optimized 3D-model also are shown and discussed. Conclusions on the applicability of the algorithm have been made.
\end{abstract}

\section{Introduction}

Products optimization after their initial creation is a follow-up process, which is a natural continuation of the development of any modern product. Construction optimizations can aim at both directions: to achieve better performance of the products and to improve the production process in terms of lower material consumption, less energy consumed, shorter production time etc., per unit of output. The optimization of production processes is particularly relevant in the context of modern requirements in terms of enterprise competitiveness, environmental protection, and depletion of natural resources. Often, satisfying these requirements is directly related to the construction of the products (or its component parts), which should be lighter to fit into a limited space, be lightweight, and have a lengthy life cycle as possible.

In most cases, parts are designed by improving on an existing design or concept, where dimensions or other design inputs are usually given as parameters. To find optimized value of these parameters, according to optimization purpose, designers typically use standard optimization methods such as design of experiments, parametric or structure optimization, etc. Sometimes, however, there are cases where the application of these methods is ineffective, as it is required to perform a number of initial and confirmatory tests (or physical experiments) while determining the optimal product design or manufacturing process conditions. That is why, with the development of so-called Computer Aided Engineering (CAE) based on numerical methods and finite element analysis (FEA), new types of optimization have been developed. In these methods, it is not necessary to pre-formulate exactly what the concept of the optimized model will be. It is enough to set the rough geometry of the existing design, the load, and the limitations in the technology to make the physical part to perform the optimization process. This allows the CAE optimization module to determine both the shape and size of each design feature. This type of optimization is also known as Topology Optimization (TO) $[1,2,3,4]$.

TO method will find the optimal distribution of material in part design, according to set of constraints defined and a given optimization goal. The algorithm works by taking a 3D Computer Aided Design (CAD) solid model in any shape and removes material from it, to minimize (or maximize) an optimization objective (which can be mass, displacement, or compliance for example) while satisfying given set of constraints, such as maximum obtained stress or displacement. Taking into account the capabilities of contemporary CAD-CAE software TO-modules, the main purpose of the present work is creating a step-by-step algorithm for optimizing the total weight of gear reducer housing elements by using TO capabilities of the contemporary CAD-CAE software. The second purpose is to study of some production controls and TO algorithm parameters effect on reaching the target reduction in weight of the gear reducer housing, and the optimization process duration.

\section{Specifics of the TO application for certain types of 3D CAD-CAE models}

Since its creation (lately 80's) until now, dozens of variations of TO methods have been developed and now many of them are integrated into most widespread

* Corresponding author: sdslavov@tu-varna.bg 
professional CAD-CAE (and even in some freeware) design software products [3]. No matter which method of TO or which of the software products (with such optimization module) will be used, the results obtained are generally quite similar [4]. As can be seen from the examples shown in Figure 1 this type of optimization can result in some very novel and complex optimized shapes (also called "organic" shape) that are often difficult to manufacture, because of the limitations of traditional manufacturing methods. In addition, in some types of parts the shape after topology optimization does not fully meet their functional purposes. This is the case with the gearbox housing shown in Figure 1, b) which in its topology optimized design, would not hold a lubricant and would not protect the gears from the negative influence of the working environment (such as exposure to dust, moisture, etc.). That is why several manufacturing controls, such as Thickness Control, Preserved Regions, Symmetry or De-Mould Controls etc., can be added into CAE TO-algorithm, in order to avoid obtaining too hollow structures or those with too jagged geometrical shape, and this way to facilitate their manufacturing [5].

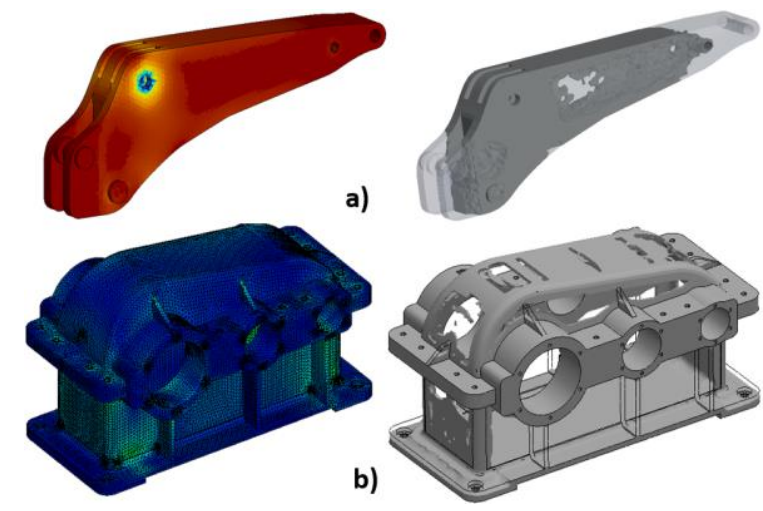

Fig. 1. Examples of topology optimized CAD designs: a) an excavator stick model; b) gear reducer housing model.

In the case shown in Figure 1, b), however, an additional post-processing work with the optimized 3D models have to be done in order to "close" the gaps in the gear reducer housing and prepare it for manufacturing by sand casting. Example of such approach is described in the source [6], where the gaps in the optimized model are enclosed with walls of lesser thickness than the original model walls. In this way the fully closed volume is preserved and the original weight of the gear reducer housing is minimised as far as possible.

The process of closing the gaps in the reducer housing element would be quite difficult if TO is performed on all model's surfaces. TO algorithm typically "omit" those 3D-model's surfaces that are set as "fixtures" or on which an external load is applied [5], but this is not always enough. There are surfaces that can be important for the proper operation of the parts or mechanisms, on which the material also should not be removed. For example, these are the surfaces of the front and rear of the excavator stick, or the bearing flanges surfaces of gearbox base and cover (see Figure 1, a, b).
So they can be set as Preserved Regions and TO algorithm will skip them from optimization process (see Figure 2, a). However, if most of the model surfaces are preserved, it may not be possible to achieve the optimization goals (for example, reaching a certain minimum mass or maximum allowed deformation). Therefore, the selection of reserved regions should be done carefully, depending on the design configuration, and the optimization goals. The manufacturing control Preserved Regions can also be used to preserve some auxiliary surfaces as shown in the Figure 2, a), that are not directly related to the product functionality, but they will facilitate the 3D-model closure process after its topology optimization. In this way, most gaps in the TO model can be easily closed by planar (or curved) shapes extruded from a closed base curves which follow the gaps contours.

Regarding the manufacturing controls used in CADCAE software products, their impacts are as follows [5]: De-mould control specifies demoulding direction of the design (see Figure 2, b). It ensures that the optimized design will be manufactural and can be extracted from a mould.
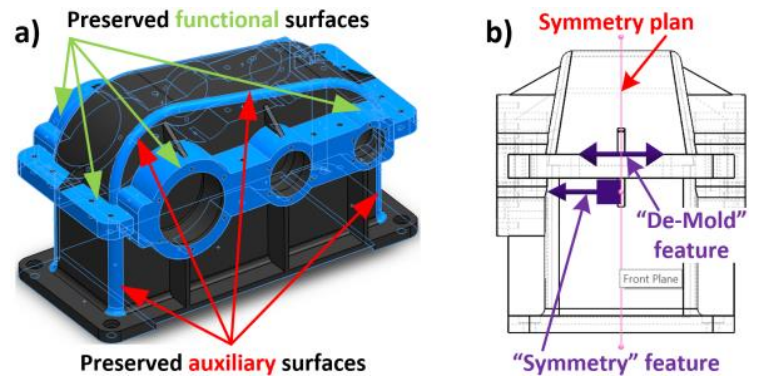

Fig. 2. a) Selected as Preserved regions surfaces in gear reducer 3D-model; b) Setting the features "Half Symmetry" and "De-Mould Control" in the 3D-model.

Thickness Control is used in TO algorithm to avoid receiving too thin walls, with a thickness less than the requirements of the manufacturing process or hollow structured models. The Symmetry Control feature (see Figure 2, b) ensures that the optimized design will be symmetric about a specified model plane (or more than one planes). Although the design of the gearbox shown in Figure 2, b) is symmetrical with respect to the vertical plane, the load as calculated values and directions of the bearing reactions is not symmetrical. Usually, in order to simplify the design of the reducer, after the gear loads are calculated, the pairs of bearings of the individual stages are chosen to be the same (based on the larger of the two calculated reactions). From this point of view, the question arises whether it is better to set a symmetrical load on the roller bearing seats or the calculated values of the individual reactions (which are generally asymmetric) to be set in the optimization study with added a Half-Symmetry Control feature.

Another important issue of the integrated TO modules in CAD-CAE software products is related with fact that only single parts or $3 \mathrm{D}$ bodies can be optimized at once [5]. TO cannot be used to simultaneously optimize assemblies or for CAD model which is made up of more than one single body. As a result, the optimization of 
more sophisticated designs made up of several parts should be done separately for each individual component. This will complicate the process, and will require preliminary analysis to determine the correct boundary conditions and interconnections of the individual parts participated in the assembly.

\section{A step-by-step algorithm for optimizing the total weight of gear reducer housing elements}

The main steps from the overall algorithm for performing TO of the gear reducer housing elements are shown in Figure 3. At the input of the algorithm some basic constructive and functional characteristics of the reducer are identified, such as transmission ratio, inclination angle of the teeth, transmitted power, input speed, the largest distance between shaft axes, etc. They are used in Step 1 for calculating loads in gears in the different reducer stages in order to determine the reactions in rolling bearings. Their calculation can be done manually, according to classical approaches from Statics and Strength of Materials [7], or by using specialized software products such as MitCalc (Czech Republic), KISSsoft AG (A Gleason Company Switzerland), etc. After clarifying the dimensions and constructive characteristics of the gears, shafts and bearings, in Step 2, the housing of the gearbox is modelled. Here some general guidance [7] in determining sidewall thickness, the thickness and width of the bearing flanges, supporting ribs, the diameters of the bolts for attaching the cover to the body or the reducer to the foundation, etc., must be taken into account. The design should also be tailored to the manufacturing method specifics (e.g. casting in sand forms). Step 2 is executed in a suitable CAD software that allows 3D modelling of the housing elements and combining them into a single body.

The next Steps 3 and 4 sets up some 3D-model important features (such as material and its mechanical properties, loads, preloaded flange and anchor bolts, etc.), and perform initial static and frequency $\mathrm{FE}$ analyses in order to test the design for mechanical strength and resistance to resonance. For more realistic analysis, it is advisable to use a Bearing load component when setting the load because this component distributes the applied bearing loads radially and non-uniformly (with sinusoidal distribution) along the cross-section of a cylindrical faces of the bearing seats [5]. If any issues with stresses, deformations, or natural frequencies after carried out FE studies are occurred, it is necessary to return in Step 2 and some changes in the initial design parameters to be made.

Step 5 sets the optimization goal by appropriate Constraints, and applicable Manufacturing controls for $\mathrm{TO}$, as it was explained in Section 2. As Constraints can be set the targeted mass that the part will be reduced, upper limit for the selected displacement component, frequency values for each mode shape, maximum permissible von Mises stress or a minimum factor of safety value for the optimized geometry, etc.
Most often, mass reduction is set as a percentage of the original mass, but can also be set as an absolute value. In Step 6, the result for the topology-optimized 3D-model of the gear reducer housing is obtained. Most of TO modules in CAD-CAE software offer possibilities for further smoothing the surfaces of the optimized 3Dmodel in order to avoid obtaining jagged edges and/or sharp angles.

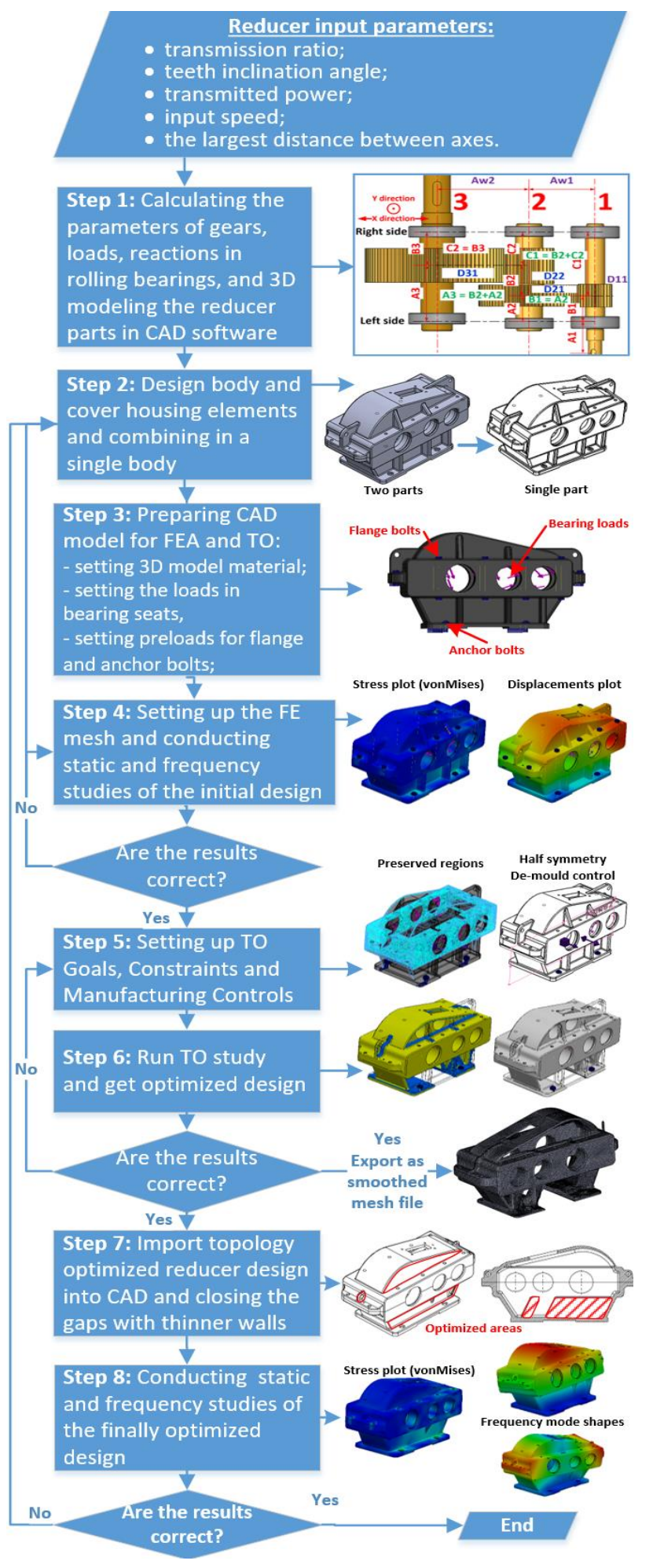

Fig. 3. Step-by-step algorithm for TO of the gear reducer housing elements. 
Sometimes Steps 5 and 6 must be repeated several times with different combinations of Constraint parameters and Manufacturing controls in order to obtain best-optimized shape of reducer housing. When it is achieved, the topology optimized 3D-model can be exported and saved as separate CAD file in the boundary geometry representation format, which is suitable for further editing and modifying.

As noted above, a topology optimized and smoothed 3D-design cannot be used directly in this state because most probably will not fully perform its functional purposes. That is why in Step 7 of the algorithm the optimized 3D-model usually is imported back into CAD software and the gaps where the material is removed after the TO are closed with walls with the smallest possible thickness (see Step 7 in the Figure 3). In addition, the considered as unnecessary housing ribs, the material around the lifting holes, the foundation flange, etc. can be entirely removed from design or reconstructed in more optimal shapes at this step also. Topology optimized 3D-model may be used as template for reconstruction of the original gear housing design so as to minimize or eliminate the material in those areas which are insignificant for ensuring its stiffness. The final 3D model can be divided again into two separate parts - the housing body and cover.

The final design of the optimized and reconstructed gear reducer housing elements must comply with all manufacture technology requirements in order to obtain a quality product. For example, if the housing elements will be manufactured by casting in sand form, a minimal thickness of the walls depending on the casting properties of the material must be respected, along with smooth transitions from thick to thin sections and large enough inclines and rounding of the wall corners [8].

In the final step 8 of the algorithm, the final optimized design is once again subjected to a static and frequency FE-study to verify that after design changes made, the optimized gear reducer housing construction do not exceed the permissible limit values. As a result of the analyses, an excess above the allowable values of the resulting stresses or deformations can be detected, or the frequency of the forced oscillation in the normal operation of the gear reducer may occur to be close to any of the natural frequencies of its design. In this case, the designer must return to one of the previous steps and make design changes or choose more appropriate options for TO process.

It should be keep in mind that topological optimization of more sophisticated or larger-scale models may take a lot of computer time. In this respect, multiple returns for resetting process parameters may make the optimization process ineffective. Moreover, it is not always clear beforehand which production controls exactly what effect they will have on the optimized construction. Therefore, the following section presents the results of a study on the effects of the model parameters and some commonly used manufacturing controls on the optimization of the housing elements of a two-stage gear reducer.

\section{Study on the effect of some model parameters and manufacturing controls over obtained TO results}

\subsection{Description of study object}

For purposes of the study housing elements from a twostage spur gear reducer shown in Figure 4, a) was used. The reducer has input operational parameters as follow:

- transmission ratio $\mathrm{i}=19.46$;

- inclination angle of the teeth $\beta=0,\left(^{\circ}\right)$ spur gears;

- transmitted power $\mathrm{P}=4.5,(\mathrm{~kW})$;

- input speed $\mathrm{n}_{1}=1500$, (rev/min),

- the largest distance between axes $\mathrm{a}_{\mathrm{w}}{ }^{\max }=90,(\mathrm{~mm})$.

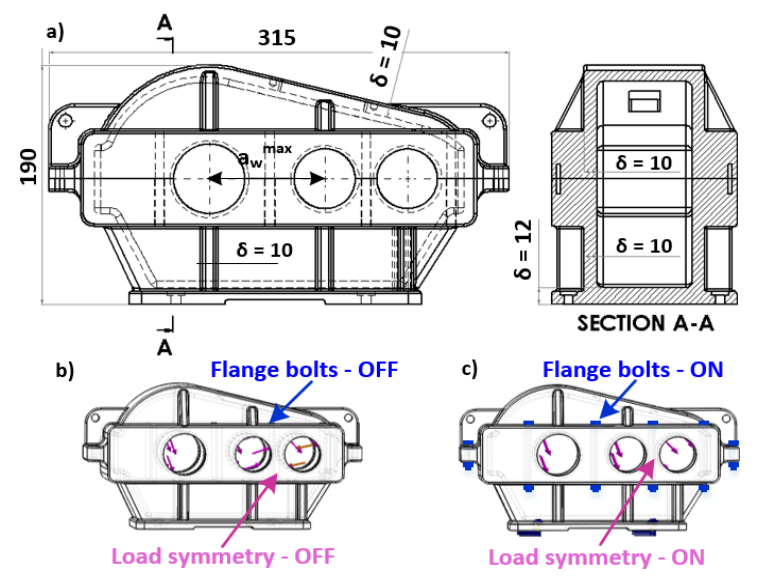

Fig. 4. a) 3D models of two-stage spur gear reducer housing elements; b) unsymmetrical bearing loads without flange bolts; c) symmetrical bearing loads with applied flange bolts;

According to them the gear loads and reactions magnitudes and directions in the bearing seats are calculated (see Table 1), together with loads in preloaded flange bolts (PFB) and preloaded anchor bolts (PAB).

Table 1. Loads applied in the reducer housing elements.

\begin{tabular}{|c|c|c|c|c|c|c|}
\hline \multirow[b]{2}{*}{$\begin{array}{c}\text { Load } \\
\text { diagrams }\end{array}$} & \multicolumn{3}{|c|}{ Left side } & \multicolumn{3}{|c|}{ Right side } \\
\hline & \multicolumn{3}{|c|}{${ }_{P A B}^{P F B}{ }_{P A B}^{R^{L}}$} & \multicolumn{3}{|c|}{ PAB } \\
\hline Reactions & $\mathbf{R} 1^{\mathrm{L}}$ & $\mathbf{R} 2^{\mathrm{L}}$ & $\mathbf{R 3}^{\mathrm{L}}$ & $R 1^{R}$ & $\mathbf{R} 2^{\mathbf{R}}$ & $\mathbf{R 3}^{\mathrm{R}}$ \\
\hline $\begin{array}{l}\text { Magni- } \\
\text { tude, (N) }\end{array}$ & 4404 & 1306 & 3157 & 807 & 5365 & 6011 \\
\hline $\begin{array}{l}\text { Direction } \\
\text { angle, (0) }\end{array}$ & $43^{\circ} 08^{\prime}$ & $109^{\circ} 43^{\prime}$ & $20^{\circ} 00^{\prime}$ & $-77^{\circ} 45^{\prime}$ & $19^{\circ} 57^{\prime}$ & $20^{\circ} 00^{\prime}$ \\
\hline PFB, (N) & \multicolumn{6}{|c|}{1200} \\
\hline $\mathrm{PAB},(\mathrm{N})$ & \multicolumn{6}{|c|}{1500} \\
\hline
\end{tabular}

Reducer housing elements are designed by using capabilities of SOLIDWORKS (SolidWorks Corp., Waltham, MA, USA) software in respect of the shape and calculated dimensions of the spur gears, shafts, and chosen rolling bearings.

Because the gear reducer housing elements are made of grey cast iron G150 (ISO 185) and is planned to be manufactured by casting in sand forms, their design is consistent with the requirements [8] of the casting process. Their total initial mass is $18.51,(\mathrm{~kg})$. The initial thickness of the body and cover walls are same for both 
of them $\delta=10,(\mathrm{~mm})$ and should not be reduced to less than $8,(\mathrm{~mm})$ in order to ensure seamless filling of the mould.

\subsection{Planning and conducting the TO-study}

In order to determine the main effects of some manufacturing controls and optimization parameters in TO process, Taguchi mixed orthogonal experimental design of the type L8 is used. This experimental design has up to four controllable factors with two levels and one factor with four levels as is shown in design section in Table 2 .

Table 2. Taguchi L8 study design and obtained results for TO of reducer housing elements.

\begin{tabular}{|c|c|c|c|c|c|c|c|}
\hline \multirow[b]{2}{*}{ } & \multicolumn{5}{|c|}{$\begin{array}{l}\text { Control factors in Taguchi mixed } \\
\text { design, type L8 }\end{array}$} & \multicolumn{2}{|c|}{$\begin{array}{c}\text { Output } \\
\text { response values }\end{array}$} \\
\hline & 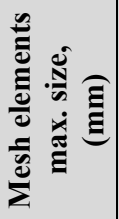 & 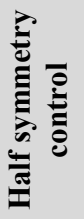 & 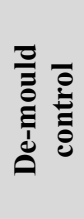 & 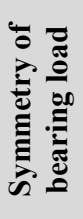 & 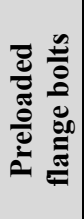 & 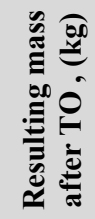 & 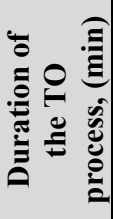 \\
\hline 1 & 3.0 & off & off & off & off & 13.37 & 515 \\
\hline 2 & 3.0 & on & on & on & on & 12.99 & 1195 \\
\hline 3 & 4.5 & off & off & on & on & 13.56 & 96 \\
\hline 4 & 4.5 & on & on & off & off & 12.99 & 143 \\
\hline 5 & 6.0 & off & on & off & on & 13.05 & 31 \\
\hline 6 & 6.0 & on & off & on & off & 13.60 & 24 \\
\hline 7 & 10.0 & off & on & on & off & 13.07 & 10 \\
\hline 8 & 10.0 & on & off & off & on & 13.94 & 5 \\
\hline
\end{tabular}

The control factors from the inner array included in the TO study (see Table 2) are as follows:

- High quality standard mesh with 4 Jacobian points is used (created by the Voronoi-Delaunay meshing scheme) and four levels of maximum element sizes: 3 , $4.5,6$, and $10,(\mathrm{~mm})$ with $\pm 5 \%$ tolerance. Total amounts of finite elements generated according to the cells size for every experimental run from Table 2 are shown in Table 3;

- Half-symmetry manufacturing control in two levels "off" and "on";

- De-mould manufacturing control in two levels - "off" and "on";

- Symmetry of bearing loads in two levels - "off" and "on". Level "off" for this factor means that calculated reactions in the bearings are set with their real magnitudes and directions in the TO model (see Figure $4, \mathrm{~b}$ ). The level "on" (see Figure 4, c) means that the biggest calculated load is set as bearing reactions (i.e. one and the same for both sides of the shafts from every gear stage).

- Preloaded flange bolts in two level - "off" and "on", where level "off" means that the preloaded flange bolts (see Table 1) are not applied, and "on" means that they are applied in the TO study.

All surfaces of the reducer body and cover flanges are selected as Preserved regions with depth 8, (mm), according Step 5 from Figure 3, which means that they do not participate in TO process.

The response parameters of the study, are respectively the resulting mass (in $\mathrm{kg}$ ) after $\mathrm{TO}$ and the duration of the optimization process (in min) (see Table 2 ). For the purpose of obtaining comparable results, all studied are carried out on the same computer system (Intel(R) Core (TM) i3 CPU, $3.2 \mathrm{GHz}$, RAM 4 GB) and by using Simulation module of SOLIDWORKS [5].

\subsection{Processing study results and discussion}

After carried out TO studies according L8 Taguchi mixed experimental design the response parameters values have been obtained (see Table 2). Resulting optimized designs of reducer housing elements are shown in the Table 3.

For processing the experimental results, Analyse Taguchi Design tool from Minitab software (Minitab Inc., State College, Pennsylvania, USA) was used. Thereby main effects are calculated and two plots of the means are generated (see Figure 5, a, b). They represented the average response for each combination of control factor levels in a static Taguchi design, and shows which factor levels minimize or maximize the response values mean.

As can be seen from Table 2 the weight of the TO gear housing designs varies from $12.99 \mathrm{~kg}$ to $13.94 \mathrm{~kg}$ or within $0.95 \mathrm{~kg}$ depending on the control factors levels.

Main effects plot for means (see Figure 5, a) shows that factors "De-mould control" and "Mesh elements size" have the greatest influence on the resultant mass variability after optimization. Expectably, the reduction

Table 3. Resulting designs of reducer housing elements after TO, according to L8 Taguchi mixed experimental design from Table 2.

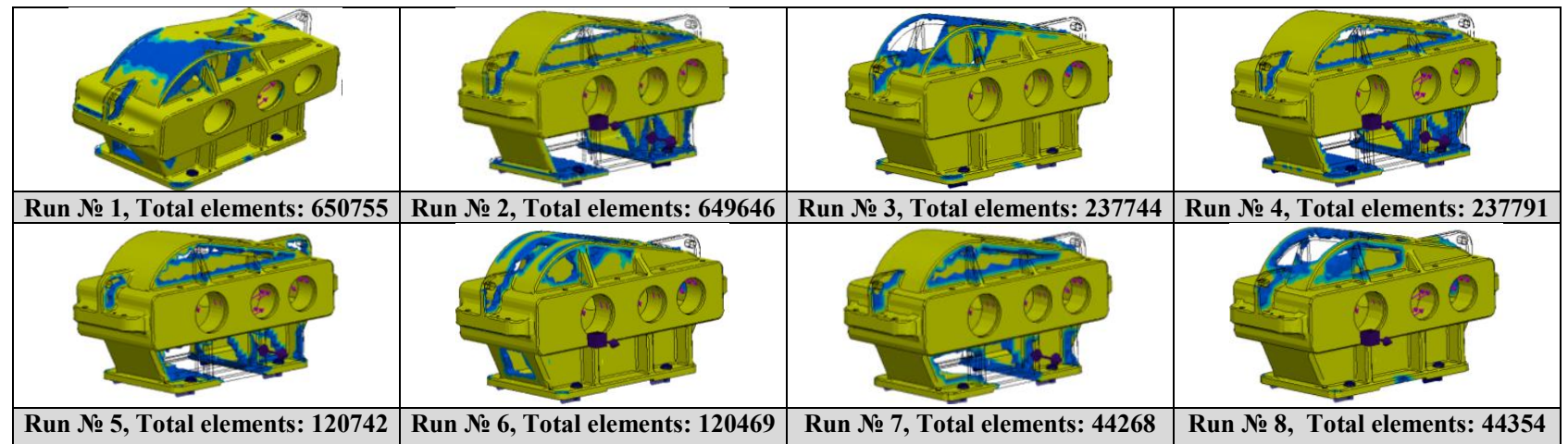


in mesh elements size leads to a decrease in the resultant mass, while substantially increasing the duration of the TO study (see Figure 5, b).

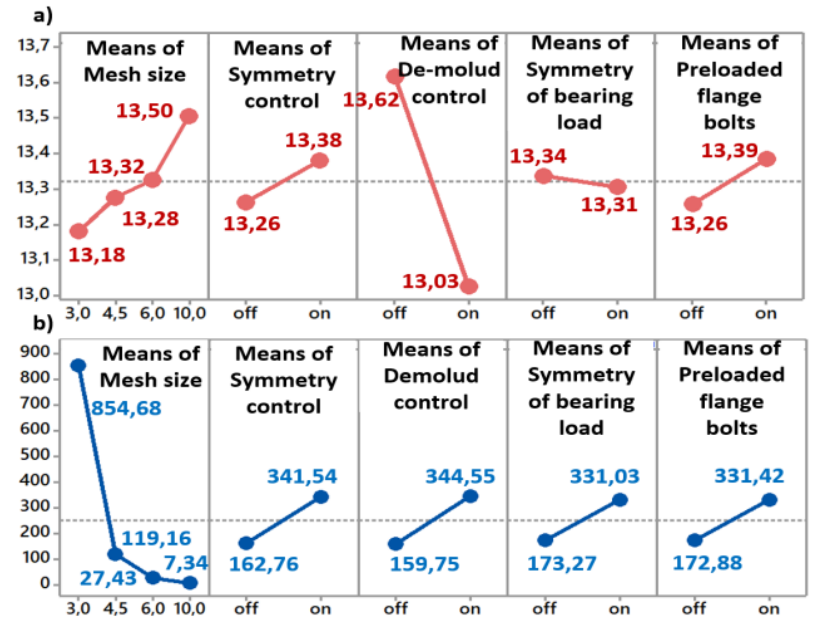

Fig. 5. Main effects plot for means: a) reducer housing elements mass (in $\mathrm{kg}$ ) after $\mathrm{TO} ; \mathrm{b}$ ) duration (in $\mathrm{min}$ ) of the $\mathrm{TO}$ process;

This behaviour is due to the fact that by reducing the size of mesh elements, the geometric shape of the 3Dmodel is better approximated, and the TO-algorithm distinguishes more precisely the elements that must remain from those that can be removed. This way however, their number increases significantly, resulting in a higher increase in optimization time. By comparing the results obtained for the optimized models (see Runs № 2, 4, 5 and 7 in the Table 2 and 3) where De-mould control is "on", against the remaining four runs $(1,3,6$ and 8) where it is "off", it can be seen that this factor mainly determines which areas of the 3D-model material will be removed. It is obvious that when this factor is switched "on", material from the surfaces which are parallel to the set direction of the De-mould is preserved, and vice versa. Thus post-processing of the gearbox housing will facilitate (see step 7 from Figure 3) because the gaps (i.e. the areas that will be closed by thinner walls) of the model will have a near to planar shape.

When "Half-symmetry control" is applied, it slightly increases the resultant mass of the 3D-model but also facilitates its post-processing because "Mirror"-function can be used in the re-design process in CAD software. If one and same loads and directions (i.e. the higher value of the calculated bearing loads $R_{i}^{L}$ or $R_{i}^{R}$ from Table 1) are used on the left and right sides of the gear housing (or "Symmetry of bearing load" factor is switched "on"), this has the least impact on mass reduction (see Figure 5, a), compared to other factors. When there are equal loads on both sides, the resulting optimized 3D-models are rather symmetrical, regardless of whether "Halfsymmetry control" is "on" or "off".

The last control factor in the Table 2 - "Preloaded flange bolts" has an expected effect on the resultant mass of the optimized model. Although the pre-loaded bolt connections in the gear housing flanges are smaller than the bearing reactions, they slightly increase the resulting mass of the housing (see Figure 5, a). Therefore, they, as well as the anchor bolts, should not be omitted when configuring the optimization algorithm.

With respect to the duration of the process, all four factors at two levels have a very similar effects (see Figure 5, b). Comparing the values obtained for TO process duration (see rows 1 and 2 in Table 2), it can be seen that if all the two-level control factors are switched "on", this leads to a doubling of the duration of TO process.

\section{Conclusion}

In the present work an algorithm in eight main steps is presented for optimization of the total weight of cast iron housing elements from gear reducers, using the topology optimization approach. All stages of the process are discussed, as well as the particularities to be respected for each of them. A study (according to Taguchi mixed orthogonal experimental design of the type L8) was carried out to investigate the impact of some TO-process parameters on the resulting optimized 3D-model.

Based on the obtained results, it can be concluded that the proposed algorithm gives satisfactory results in the optimization of reducer housing elements weight of the examined type and configuration. By using the right combinations of TO parameters and manufacturing controls, weight reduction between 25 to $30 \%$ can be achieved, as well as simplifying the post-processing of optimized 3D-models to their final design.

\section{References}

1. Bendsøe, M.P. Structural Optimization (1989) 1: 193. https://doi.org/10.1007/BF01650949

2. Harzheim, L. \& Graf, G. Struct Multidisc Optim (2006) 31: 388. https://doi.org/10.1007/s00158-0050554-9

3. Topology Optimization Guide. Available online: http://www.topology-opt.com/software-list/ (last visited 08.02.2019)

4. Deaton, Joshua D., and Ramana V. Grandhi. "A survey of structural and multidisciplinary continuum topology optimization: post 2000." Structural and Multidisciplinary Optimization 49.1 (2014): 1-38.

5. SOLIDWORKS Simulation Help. Available online: http://help.solidworks.com/2018/english/solidworks/ cworks/idc help helptopics.htm. (last visited 08.03.2019).

6. Slavov S, Konsulova-Bakalova M. Optimizing Weight of Housing Elements of Two-stage Reducer by Using the Topology Management Optimization Capabilities Integrated in SOLIDWORKS: A Case Study. Machines. 2019; 7(1):9

7. Bhandari, V.B. Design of Machine Elements; Tata McGraw-Hill Education: New York, NY, USA, 2010.

8. Stefanescu, D.M. Science and Engineering of Casting Solidification, 2nd ed.; Springer: Berlin/Heidelberg, Germany, 2008 